\title{
Global attractiveness and exponential stability for impulsive fractional neutral stochastic evolution equations driven by $\mathrm{fBm}$
}

Jiankang Liu' ${ }^{1,2}$, Wei Xu ${ }^{1 *}$ (D) and Qin Guo

"Correspondence:

weixu@nwpu.edu.cn

${ }^{1}$ Department of Applied

Mathematics, Northwestern

Polytechnical University, Xi'an, China

Full list of author information is

available at the end of the article

\section{Springer}

\begin{abstract}
This paper is concerned with a class of fractional neutral stochastic integro-differential equations with impulses driven by fractional Brownian motion (fBm). First, by means of the resolvent operator technique and contraction mapping principle, we can directly show the existence and uniqueness result of mild solution for the aforementioned system. Then we develop a new impulsive-integral inequality to obtain the global attracting set and pth moment exponential stability for this type of equation. Worthy of note is that this powerful inequality after little modification is applicable to the case with delayed impulses. Moreover, sufficient conditions which guarantee the pth moment exponential stability for some pertinent systems are stated without proof. In the end, an example is worked out to illustrate the theoretical results.
\end{abstract}

Keywords: Global attracting set; Exponential stability; Fractional neutral stochastic integro-differential equations; Caputo fractional derivative; Delayed impulses

\section{Introduction}

In recent two decades, fractional stochastic evolution equations have grabbed the attention of many researchers, owing to their applications in various fields, such as physics, chemistry, viscoelasticity, heat conduction, aerodynamics, electrodynamics of complex medium, electricity mechanics, and so forth (see, e.g., [1-4] and the references therein). Meanwhile, impulsive dynamical systems arise as a natural characterization of these realworld phenomena and processes which are frequently subject to instantaneous perturbations and experience abrupt changes (impulses). For details on theory and applications of such systems, we refer to the monographs $[5,6]$, the papers $[7,8]$ etc. and the references therein. Moreover, considering that the evolution of abundant dynamical systems depends not only on current state and past state but also upon the derivative of the history state, many authors have studied impulsive fractional neutral stochastic evolution equations (IFNSEEs). The accumulated results mainly focus on well-posedness, stability and controllability; for instance, see [9-11] and the references therein.

On the other hand, along with the research of stability (see, e.g., $[7-9,12,13]$ and the references therein), there has been an increasing interest in study on global attracting sets

(c) The Author(s) 2020. This article is licensed under a Creative Commons Attribution 4.0 International License, which permits use, sharing, adaptation, distribution and reproduction in any medium or format, as long as you give appropriate credit to the original author(s) and the source, provide a link to the Creative Commons licence, and indicate if changes were made. The images or other third party material in this article are included in the article's Creative Commons licence, unless indicated otherwise in a credit line to the material. If material is not included in the article's Creative Commons licence and your intended use is not permitted by statutory regulation or exceeds the permitted use, you will need to obtain permission directly from the copyright holder. To view a copy of this licence, visit http://creativecommons.org/licenses/by/4.0/. 
of stochastic dynamical systems over the last few years, since attracting sets guarantee the solutions enter some sets and do not exist. Among others, motivated by Chen's work [13], Long et al. [14] examined the global attractiveness and exponential stability of impulsive stochastic neutral evolution equations driven by Q-Wiener process. Li [15] discussed the global attracting set and quasi-invariant set of impulsive neutral stochastic functional partial differential equations driven by fractional Brownian motion $(\mathrm{fBm})$, where the Hurst exponent $H \in\left(\frac{1}{2}, 1\right)$. Also, the global attracting set of the neutral stochastic partial differential equation with finite delay and additive $\alpha$-stable process is given by Liu and Li [16]. Duan and ren [17] proved the attractiveness and quasi-invariant set of impulsive neutral stochastic integro-differential equation driven by $\mathrm{fBm}$ with Hurst parameter $\frac{1}{2}<H<1$. In view of the practicability of second-order stochastic differential equations (see, e.g., [18]), $\mathrm{Xu}$ et al. [19] showed global attracting sets of second-order neutral stochastic evolution equations driven by a $\mathrm{fBm}$ and an independent standard Wiener process, without considering impulsive effects. Here it is worth noting that Deng and Shu [20] established an impulsive-integral inequality to obtain the exponential stability of mild solution to impulsive neutral stochastic functional differential equations driven by $\mathrm{fBm}$ with noncompact semigroup. Different from the papers mentioned above, Xu and Luo [21] derived the global attracting set and exponential stability of neutral stochastic evolution equations driven by fBm with the Hurst exponent $H \in\left(0, \frac{1}{2}\right)$. Nevertheless, the aforementioned papers cannot answer the question that if the first or the second-order derivative is replaced by a fractional-order derivative. That is to say, there is no study on global attracting sets and exponential stability of impulsive fractional neutral stochastic evolution equations driven by $\mathrm{fBm}$ in the existing literature. Moreover, in some real-world problems, the impulsive functions depend on former state variables [22], i.e., $\Delta x\left(t_{k}\right)=I_{k}\left(x_{t_{k}}\right)$. All these facts push us to develop and explore techniques and methods for the global attractiveness and exponential stability of IFNSEEs, especially with delayed impulses.

Based on the above statement and analysis, this work will be devoted to deriving the global attracting set and exponential stability for IFNSEEs. We use the resolvent operator technique, inequality technique and stochastic analysis theory to attain this goal. In addition, we summarize and highlight the contributions of this paper as follows.

- The global attracting set and exponential stability of a class of fractional neutral stochastic integro-differential equations with impulses driven by fBm are obtained.

- We establish a new impulsive-integral inequality, and this key inequality is applicable to investigate the global attracting set and exponential stability for various types of neutral stochastic evolution equations with delayed impulses.

- By the established impulsive-integral inequality, we develop the $p$ th moment exponential stable results of some pertinent systems without proof.

The rest of this paper is organized as follows. Section 2 introduces some preliminary notations, lemmas, hypotheses, definitions and the mathematical model. Section 3 focuses on proposing a new impulsive-integral inequality which is a key tool in proving the global attracting set and exponential stability of mild solutions for IFNSEEs. Some useful remarks are also discussed. In Sect. 4, the global attracting set of the considered system is proved and exponential stable results of the concerned system and relevant systems are presented. In Sect. 5, an example is provided to illustrate the obtained theoretical results. Finally, we give the conclusion of this paper in Sect. 6. 


\section{Preliminary}

$(\Omega, \mathcal{F}, \mathbb{P})$ stands for a complete probability space equipped with a normal filtration $\left\{\mathcal{F}_{t}\right\}_{t \geq 0}$ satisfying the usual conditions. Let $\mathbb{H}, \mathbb{K}$ be two real, separable Hilbert spaces and $L(\mathbb{K}, \mathbb{H})$ represents the family of all bounded linear operators from $\mathbb{K}$ to $\mathbb{H}$. For simplicity, we use the same notation $\|\cdot\|$ to express the norms in $\mathbb{H}$, $\mathbb{K}$ and $L(\mathbb{K}, \mathbb{H})$. Let $\mathbb{K}_{0}=\mathcal{Q}^{\frac{1}{2}} \mathbb{K}$, and $L_{2}^{0}=L_{2}\left(\mathbb{K}_{0}, \mathbb{H}\right)$ be a separable Hilbert space of all Hilbert-Schmidt operators from $\mathcal{Q}^{\frac{1}{2}} \mathbb{K}$ to $\mathbb{H}$ with the norm $\|\cdot\|_{L_{2}^{0}} \cdot \mathcal{P C}([-r, 0] ; \mathbb{H})$ indicates the space of all càdlàg functions $\psi$ from $[-r, 0]$ to $\mathbb{H}$, and for $\psi(t) \in \mathcal{P C},\|\psi\|_{\mathcal{P C}}=\sup _{-r \leq t \leq 0}\|\psi(t)\|$.

$Q \in L(\mathbb{K}, \mathbb{K})$ denotes a non-negative self-adjoint operator and let $L_{Q}^{0}(\mathbb{K}, \mathbb{H})$ be the space of all $\xi \in L(\mathbb{K}, \mathbb{H})$ such that $\xi Q^{\frac{1}{2}}$ is a Hilbert-Schmidt operator endowed with the norm $\|\xi\|_{L_{Q}^{0}(\mathbb{K}, \mathbb{H})}^{2}=\operatorname{tr}\left(\xi Q \xi^{*}\right)$. Consider the following series:

$$
B_{Q}^{H}(t)=\sum_{n=1}^{\infty} \beta_{n}^{H}(t) Q^{\frac{1}{2}} e_{n}, \quad t \geq 0
$$

where $\left\{\beta_{n}^{H}(t)\right\}_{n \in \mathbb{N}}$ is a sequence of two-sided one-dimensional standard fBm mutually independent on $(\Omega, \mathcal{F}, \mathbb{P})$ and $\left\{e_{n}\right\}_{n \in \mathbb{N}}$ is a complete orthonormal basis in $\mathbb{K}$. This series converges in the space $\mathbb{K}$ when $Q$ is a non-negative self-adjoint trace class operator, namely, $B_{Q}^{H}(t) \in L^{2}(\Omega, \mathbb{K})$. Then the above $B_{Q}^{H}(t)$ is called a $\mathbb{K}$-valued $Q$-cylindrical fBm with covariance operator $Q$. For example, if $\left\{\lambda_{n}\right\}_{n \in \mathbb{N}}$ is a bounded sequence of non-negative real numbers such that $Q e_{n}=\lambda_{n} e_{n}$, supposing that $Q$ is a nuclear operator in $\mathbb{K}$ (that is, $\left.\sum_{n=1}^{\infty} \lambda_{n}<\infty\right)$, then the $\mathbb{K}$-valued $Q$-cylindrical $\mathrm{fBm}$

$$
B_{Q}^{H}(t)=\sum_{n=1}^{\infty} \beta_{n}^{H}(t) Q^{\frac{1}{2}} e_{n}=\sum_{n=1}^{\infty} \sqrt{\lambda_{n}} \beta_{n}^{H}(t) e_{n}, \quad t \geq 0
$$

is well-defined. For more details on $\mathrm{fBm} B_{Q}^{H}(t)$ and the stochastic integral with respect to $\mathrm{fBm} B_{Q}^{H}(t)$, one can refer to [23, 24].

In this paper, we discuss the impulsive fractional neutral stochastic integro-differential equation driven by $\mathrm{fBm}$ of the following form:

$$
\left\{\begin{aligned}
&{ }^{c} D_{t}^{\alpha}\left[x(t)-g\left(t, x_{t}\right)\right]= A\left[x(t)-g\left(t, x_{t}\right)\right]+\mathbb{I}_{t}^{2-\alpha}\left(f\left(t, x_{t}\right)\right) \\
& \quad+h\left(t, x_{t}\right) \frac{d W(t)}{d t}+\sigma(t) \frac{d B_{Q}^{H}(t)}{d t}, \quad t \neq t_{k}, t \in J, \\
& \Delta x\left(t_{k}\right)=I_{k}\left(x\left(t_{k}^{-}\right)\right), \quad \Delta x^{\prime}\left(t_{k}\right)=G_{k}\left(x\left(t_{k}^{-}\right)\right), \quad t=t_{k}, k=1,2, \ldots, \\
& x(t)=\psi(t) \in \mathcal{P C}_{\mathcal{F}_{0}}^{b}([-r, 0] ; \mathbb{H}), \quad t \in[-r, 0], \\
& \frac{d}{d t}\left[x(t)-g\left(t, x_{t}\right)\right]_{t=0}=\varphi \in \mathbb{H},
\end{aligned}\right.
$$

where ${ }^{c} D_{t}^{\alpha}$ is the fractional derivative of order $\alpha \in(1,2)$ in the sense of Caputo, $A: \mathcal{D}(A) \subseteq$ $\mathbb{H} \rightarrow \mathbb{H}$ is a densely defined closed linear operator, $\mathbb{I}_{t}^{\alpha}$ denotes the $\alpha$ th order fractional integral, the functions $g, f: J \times \mathcal{P C} \rightarrow \mathbb{H}, h: J \times \mathcal{P C} \rightarrow L_{2}^{0}, \sigma: J \rightarrow L_{Q}^{0}(\mathbb{K}, \mathbb{H}), I_{k}, G_{k}: \mathcal{P C} \rightarrow \mathbb{H}$ are appropriate continuous functions, the history process $x_{t}=x(t+\tau),-r \leq \tau \leq 0$ is $\mathcal{P C}$ valued, $B_{Q}^{H}(t)$ denotes a fractional Brownian motion with Hurst exponent $H \in\left(\frac{1}{2}, 1\right), W(t)$ symbolizes a standard Wiener process independent of $B_{Q}^{H}(t) . \mathcal{P} \mathcal{C}_{\mathcal{F}_{0}}^{b}([-r, 0] ; \mathbb{H})$ represents the family of all almost surely bounded, $\mathcal{F}_{0}$-measurable, $\mathcal{P C}$-valued random variables. Moreover, $\Delta x\left(t_{k}\right)=x\left(t_{k}^{+}\right)-x\left(t_{k}^{-}\right), \Delta x^{\prime}\left(t_{k}\right)=x^{\prime}\left(t_{k}^{+}\right)-x^{\prime}\left(t_{k}^{-}\right), x\left(t_{k}^{+}\right)$and $x\left(t_{k}^{-}\right)$stand for the right 
and left limits of $x(t)$ at $t_{k}, x^{\prime}\left(t_{k}^{+}\right)$and $x^{\prime}\left(t_{k}^{-}\right)$indicate the right and left limits of $x^{\prime}(t)$ at $t_{k}$, and the fixed impulsive sequence $t_{k}$ satisfies $0<t_{1}<\cdots<t_{k}<t_{k+1}<\cdots$ with $\lim _{k \rightarrow \infty} t_{k}=\infty$.

Before starting the analyses, we state some basic definitions and introduce the required lemmas.

Definition 2.1 ([1]) For the function $f \in L^{1}((0, T), H), 0<T<\infty$, the Riemann-Liouville fractional integral of $f$ of order $\alpha>0$ is given as

$$
\mathrm{RL}_{\mathbb{I}_{t}^{\alpha}} f(t)=\frac{1}{\Gamma(\alpha)} \int_{0}^{t}(t-s)^{\alpha-1} f(s) d s
$$

Definition 2.2 ([1]) For the function $f \in L^{1}((0, T), \mathbb{H})$, the fractional derivative of $f$ of order $\alpha$ in the Riemann-Liouville sense is given by

$$
{ }^{\mathrm{RL}} D_{t}^{\alpha} f(t)=\frac{d^{m}}{d t^{m}} \mathbb{I}_{t}^{m-\alpha} f(t), \quad m-1<\alpha<m, m \in \mathbb{N}^{+},
$$

where ${ }^{\mathrm{RL}} \mathbb{I}_{t}^{m-\alpha} f \in \mathrm{Z}^{m, 1}((0, T), \mathbb{H}) . \mathrm{Z}^{m, 1}((0, T), \mathbb{H})$ is the Sobolev space defined by

$$
\begin{aligned}
\mathrm{Z}^{m, 1}((0, T), \mathbb{H})= & \left\{x \in \mathbb{H}, \exists w \in L^{1}((0, T), \mathbb{H}): x(t)=\sum_{k=0}^{m-1} e_{k} \frac{t^{k}}{k !}\right. \\
& \left.+\frac{t^{m-1}}{(m-1) !} * w(t)\right\},
\end{aligned}
$$

where $w(t)=x^{m}(t), e_{k}=x^{k}(0)$.

Definition 2.3 ([1]) For the function $f \in C^{m-1}((0, T), \mathbb{H}) \cap L^{1}((0, T), \mathbb{H})$, the fractional derivative of $f$ of order $\alpha$ in the Caputo sense is defined by

$$
{ }^{c} D_{t}^{\alpha} f(t)=\frac{1}{\Gamma(m-\alpha)} \int_{0}^{t}(t-s)^{m-\alpha-1} f^{m}(s) d s, \quad m-1<\alpha<m .
$$

Definition 2.4 ([25]) Let $A: \mathcal{D}(A) \subseteq \mathbb{H} \rightarrow \mathbb{H}$ be a closed linear operator. Then $A$ is called a $(M, \theta, \alpha, \mu)$-type sectorial operator if there exist three constants $\mu \in \mathbb{R}, \theta \in\left(0, \frac{\pi}{2}\right)$ and $M>0$ such that

(a) The $\alpha$-resolvent of $A$ exists an exterior of the sector

$$
\mu+S_{\theta}=\left\{\mu+\lambda^{\alpha}: \lambda \in \mathbb{C},\left|\operatorname{Arg}\left(-\lambda^{\alpha}\right)\right|<\theta\right\},
$$

(b) $\left\|R\left(\lambda^{\alpha}, A\right)\right\|=\left\|\left(\lambda^{\alpha} I-A\right)^{-1}\right\| \leq \frac{M}{\left|\lambda^{\alpha}-\mu\right|}, \lambda^{\alpha} \notin \mu+S_{\theta}$.

Lemma 2.5 ([26]) Assume that $A$ is a $(M, \theta, \alpha, \mu)$-type sectorial operator, and f fulfills the uniform Hölder condition with index $\gamma \in(0,1]$, then the unique solution of the following Cauchy problem:

$$
\left\{\begin{array}{l}
{ }^{c} D_{t}^{\alpha} x(t)=A x(t)+f(t), \quad 1<\alpha<2, t \in[0, T] \\
x(0)=x_{0} \in \mathbb{H}, \quad x^{\prime}(0)=x_{1} \in \mathbb{H},
\end{array}\right.
$$


is given as

$$
x(t)=\mathcal{S}_{\alpha}(t) x_{0}+\mathcal{K}_{\alpha}(t) x_{1}+\int_{0}^{t} \mathcal{T}_{\alpha}(t-s) f(s) d s .
$$

Note that the operators $\mathcal{S}_{\alpha}(t), \mathcal{K}_{\alpha}(t), \mathcal{T}_{\alpha}(t)$ are determined by

$$
\begin{aligned}
& \mathcal{S}_{\alpha}(t)=\frac{1}{2 \pi i} \int_{C} e^{\lambda t} \lambda^{\alpha-1} R\left(\lambda^{\alpha}, A\right) d \lambda, \\
& \mathcal{K}_{\alpha}(t)=\frac{1}{2 \pi i} \int_{C} e^{\lambda t} \lambda^{\alpha-2} R\left(\lambda^{\alpha}, A\right) d \lambda, \\
& \mathcal{T}_{\alpha}(t)=\frac{1}{2 \pi i} \int_{C} e^{\lambda t} R\left(\lambda^{\alpha}, A\right) d \lambda
\end{aligned}
$$

where $C$ is a suitable path with $\lambda^{\alpha} \notin \mu+S_{\theta}, \lambda \in \mathbb{C}$.

Then, following Theorem 4.3 in Ref. [26] and Lemma 2.1, Definition 3.1 in Ref. [27], we introduce the definition of mild solution to the considered system (1).

Definition 2.6 An $\mathbb{H}$-valued stochastic process $x(t)$ is said to be a mild solution to Eq. (1), if

(a) $x(t)$ is $\mathcal{F}_{t}$-adapted for each $t \in[0, T]$, with $\mathbb{P}\left\{\omega: \int_{0}^{T}\|x(t)\|^{2} d t<\infty\right\}=1$ almost surely;

(b) $x(t) \in \mathbb{H}, 0 \leq t \leq T$ has càdlàg paths a.s., and for arbitrary $t \in[0, T], x(t)$ satisfies the integral equation as follows:

$$
\begin{aligned}
x(t)= & \mathcal{S}_{\alpha}(t)[\psi(0)-g(0, \psi)]+\mathcal{K}_{\alpha}(t) \varphi+g\left(t, x_{t}\right)+\int_{0}^{t} \mathcal{K}_{\alpha}(t-s) f\left(s, x_{s}\right) d s \\
& +\int_{0}^{t} \mathcal{T}_{\alpha}(t-s) h\left(s, x_{s}\right) d W(s)+\int_{0}^{t} \mathcal{T}_{\alpha}(t-s) \sigma(s) d B_{Q}^{H}(s) \\
& +\sum_{0<t_{k}<t} \mathcal{S}_{\alpha}\left(t-t_{k}\right) I_{k}\left(x\left(t_{k}^{-}\right)\right)+\sum_{0<t_{k}<t} \mathcal{K}_{\alpha}\left(t-t_{k}\right) G_{k}\left(x\left(t_{k}^{-}\right)\right), \\
x(t)= & \psi(t) \in \mathcal{P C}_{\mathcal{F}_{0}}^{b}([-r, 0] ; \mathbb{H}), \quad t \in[-r, 0] .
\end{aligned}
$$

Definition 2.7 ([14]) The set $\mathbb{S} \subset \mathbb{H}$ is called a global attracting set for Eq. (1), if for any initial data $\psi \in \mathcal{P} \mathcal{C}_{\mathcal{F}_{0}}^{b}$, the solution $x_{t}(0, \psi)$ converges to $\mathbb{S}$ as $t \rightarrow+\infty$ i.e.,

$$
\operatorname{dist}\left(x_{t}(0, \psi), \mathbb{S}\right) \rightarrow 0 \quad \text { as } t \rightarrow+\infty \text {, }
$$

where $\operatorname{dist}(x, \mathbb{S})=\inf _{y \in S} \mathbb{E}(\|x-y\|)$.

Definition 2.8 ([14]) The mild solution $x(t)$ for system (1) with initial condition $\psi \in \mathcal{P} \mathcal{C}_{\mathcal{F}_{0}}^{b}$ is said to be $p$ th moment exponentially stable if there exist a pair of constants $\mathbb{M} \geq 1$ and $\eta>0$ such that

$$
\mathbb{E}\|x(t)\|^{p} \leq \mathbb{M} e^{-\eta t}, \quad t \geq 0, p \geq 2
$$


Lemma 2.9 ([28]) Suppose that $\Psi(t), t \geq 0$ is a $L_{2}^{0}$-valued predictable process, then, for any arbitrary $p \geq 2$, there exists a constant $C_{p}>0$ such that

$$
\sup _{t \in[0, T]} \mathbb{E}\left\|\int_{0}^{t} \Psi(s) d W(s)\right\|^{p} \leq C_{p}\left(\int_{0}^{T}\left(\mathbb{E}\|\Psi(s)\|_{L_{2}^{0}}^{p}\right)^{2 / p} d s\right)^{p / 2},
$$

where $C_{p}=(p(p-1) / 2)^{p / 2}$.

Lemma 2.10 ([28]) Let $p \geq 2$ and $x, y \in \mathbb{H}$, then, for any $\delta \in(0,1]$,

$$
\begin{aligned}
& \|x+y\|^{p} \leq(1+\delta)^{p-1}\|x\|^{p}+(1+1 / \delta)^{p-1}\|y\|^{p}, \\
& \|x+y\|^{p} \leq\|x\|^{p} / \delta^{p-1}+\|y\|^{p} /(1-\delta)^{p-1} .
\end{aligned}
$$

So as to achieve the desired goals, we impose the following hypotheses for the concerned system (1).

(H1) The $\alpha$-resolvent family $\mathcal{S}_{\alpha}(t), \mathcal{K}_{\alpha}(t), \mathcal{T}_{\alpha}(t)$ generated by the sectorial operator $A$ is compact on $\overline{\mathcal{D}(A)}$, and for all $t \geq 0$, there exist four constants $\widetilde{M} \geq 1$ and $\gamma_{1}, \gamma_{2}, \gamma_{3}>$ 0 such that

$$
\left\|\mathcal{S}_{\alpha}(t)\right\| \leq \tilde{M} e^{-\gamma_{1} t}, \quad\left\|\mathcal{K}_{\alpha}(t)\right\| \leq \tilde{M} e^{-\gamma_{2} t}, \quad\left\|\mathcal{T}_{\alpha}(t)\right\| \leq \widetilde{M} e^{-\gamma_{3} t}
$$

(H2) (a) The function $g(t, \cdot) \in \mathcal{D}\left((-A)^{\beta}\right)$ for $\beta \in(0,1]$ and the mapping $(-A)^{\beta} g(t, \cdot)$ is globally Lipschitz continuous, i.e., for $\forall \xi, \eta \in \mathcal{P C}, t \geq 0$, there exists a positive constant $k_{0}$ such that

$$
\left\|(-A)^{\beta} g(t, \xi)-(-A)^{\beta} g(t, \eta)\right\| \leq k_{0}\|\xi-\eta\|_{\mathcal{P C}},
$$

and $g(t, 0)=0$, the inequality $\delta=\left\|(-A)^{-\beta}\right\| k_{0}<1$ holds.

(b) The operator $\mathrm{P}: \mathcal{P C}([-r, T], \mathbb{H}) \rightarrow \mathcal{P C}([0, T], \mathbb{H})$ is a completely continuous operator defined by $\mathrm{P} x_{t}=\frac{d}{d t}\left(g\left(t, x_{t}\right)\right)$ such that the set $\left\{\mathrm{P} x_{t}: x_{t} \in \mathcal{P C}([-r, T], \mathbb{H}), t \in\right.$ $[0, T]\}$ is precompact in $\mathbb{H}$.

(H3) The coefficient functions $f(t, \cdot), h(t, \cdot)$ are globally Lipschitz continuous, that is, for $\forall \xi, \eta \in \mathcal{P C}$, there exist positive constants $C_{f}, C_{h}, l_{f}, l_{h}$ such that

$$
\begin{aligned}
& \|f(t, \xi)-f(t, \eta)\| \leq C_{f}\|\xi-\eta\|_{\mathcal{P C}}, \quad\|f(t, 0)\| \leq l_{f}, \\
& \|h(t, \xi)-h(t, \eta)\|_{L_{2}^{0}} \leq C_{h}\|\xi-\eta\|_{\mathcal{P C}}, \quad\|h(t, 0)\|_{L_{2}^{0}} \leq l_{h},
\end{aligned}
$$

for all $t \geq 0$.

(H4) The function $\sigma:[0,+\infty) \rightarrow L_{Q}^{0}(\mathbb{K}, \mathbb{H})$ satisfies for the complete orthonormal basis $\left\{e_{n}\right\}_{n \in N^{+}} \in \mathbb{K}$, the condition that

$$
\begin{aligned}
& \sum_{n=1}^{\infty}\left\|\sigma(t) Q^{\frac{1}{2}} e_{n}\right\|_{L^{2}([0, T] ; \mathbb{H})}<\infty \\
& \sum_{n=1}^{\infty}\left\|\sigma(t) Q^{\frac{1}{2}} e_{n}\right\| \text { is uniformly convergent for } t \in[0, T] .
\end{aligned}
$$


(H5) For every $k=1,2, \ldots$, there exist positive constants $c_{k}, d_{k}$ for all $x, y \in \mathbb{H}$ such that

$$
\begin{aligned}
& \left\|I_{k}(x)-I_{k}(y)\right\| \leq c_{k}\|x-y\|, \quad\left\|I_{k}(0)\right\|=0, \\
& \left\|G_{k}(x)-G_{k}(y)\right\| \leq d_{k}\|x-y\|, \quad\left\|G_{k}(0)\right\|=0,
\end{aligned}
$$

and $\sum_{k=1}^{\infty} c_{k}, \sum_{k=1}^{\infty} d_{k}<+\infty$.

Remark 2.11 According to the estimates on $\left\|\mathcal{S}_{\alpha}(t)\right\|,\left\|\mathcal{K}_{\alpha}(t)\right\|,\left\|\mathcal{T}_{\alpha}(t)\right\|$ in Ref. [25], we know they are not only bounded, but tend to zero as $t \rightarrow \infty$ when $\mu<0$ and given $\phi \in(0, \pi)$. So combining with the work of Ref. [27], Hypothesis $(H 1)$ is justified.

Under assumptions $(H 1)-(H 5)$, by virtue of inequality techniques and the Banach fixed point theorem, one can obtain the existence and uniqueness of mild solution for system (1) on $[-r, T], 0<T<\infty$ without difficulty.

\section{Impulsive-integral inequality}

In this part, we prove the following impulsive-integral inequality which will be used to obtain the global attracting set and $p$ th moment exponential stability of system (1).

Lemma 3.1 Assume that $y(t):[-r,+\infty) \rightarrow \mathbb{R}^{+}$is a solution of the delay integral inequality:

$$
y(t) \leq \begin{cases}a_{1} e^{-\gamma_{1} t}+a_{2} e^{-\gamma_{2} t}+a_{3}\left\|y_{t}\right\|_{\mathcal{P C}}+a_{4} \int_{0}^{t} e^{-\gamma_{2}(t-s)}\left\|y_{s}\right\|_{\mathcal{P C}} d s & \\ \quad+a_{5} \int_{0}^{t} e^{-\gamma_{3}(t-s)}\left\|y_{s}\right\|_{\mathcal{P C}} d s+\sum_{0<t_{k}<t} c_{k} e^{-\gamma_{1}\left(t-t_{k}\right)} y\left(t_{k}^{-}\right) & \\ \quad+\sum_{0<t_{k}<t} d_{k} e^{-\gamma_{2}\left(t-t_{k}\right)} y\left(t_{k}^{-}\right)+a_{6}, & t \geq 0, \\ \psi(t), & t \in[-r, 0],\end{cases}
$$

where $\psi(t) \in \mathcal{P C}\left([-r, 0] ; \mathbb{R}^{+}\right), \gamma_{1}, \gamma_{2}, \gamma_{3}>0$ and $a_{i}(i=1,2, \ldots, 6), c_{k}, d_{k}(k=1,2, \ldots)$ are non-negative constants. If

$$
a_{3}+\frac{a_{4}}{\gamma_{2}}+\frac{a_{5}}{\gamma_{3}}+\sum_{k=1}^{\infty} c_{k}+\sum_{k=1}^{\infty} d_{k} \stackrel{\Delta}{=} \kappa<1
$$

then we have

$$
y(t) \leq K e^{-\gamma t}+\frac{a_{6}}{1-\kappa}, \quad \forall t \geq 0,
$$

where $\gamma \in\left(0, \gamma^{*}\right), \gamma^{*}=\min \left\{\gamma_{1}, \gamma_{2}, \gamma_{3}\right\}, K \geq\|\psi\|_{\mathcal{P C}}$ and

$$
\frac{a_{1}+a_{2}}{K}+a_{3} e^{\gamma r}+\frac{a_{4} e^{\gamma r}}{\gamma_{2}-\gamma}+\frac{a_{5} e^{\gamma r}}{\gamma_{3}-\gamma}+\sum_{k=1}^{\infty} c_{k}+\sum_{k=1}^{\infty} d_{k}<1
$$

Proof First we remark that once the inequality (3), i.e., $\kappa<1$ is determined, there exist constants $\gamma \in\left(0, \gamma^{*}\right), K \geq\|\psi\|_{\mathcal{P C}}$ such that Eq. (4) is workable. Next, we prove the statement by contradiction. If the assertion of Lemma 3.1 was false, there must be a $\widehat{t}>0$ such that

$$
y(\widehat{t}) \geq K e^{-\gamma \widehat{t}}+\frac{a_{6}}{1-\kappa}
$$


and

$$
y(t)<K e^{-\gamma t}+\frac{a_{6}}{1-\kappa}, \quad t \in[-r, \widehat{t}] .
$$

In view of (2) and (6), we derive

$$
\begin{aligned}
& y \widehat{t}) \leq a_{1} e^{-\gamma_{1} \widehat{t}}+a_{2} e^{-\gamma_{2} \widehat{t}}+a_{3}\left\|y_{\hat{t}}\right\|_{\mathcal{P C}}+a_{4} \int_{0}^{\widehat{t}} e^{-\gamma_{2}(\hat{t}-s)}\left\|y_{s}\right\|_{\mathcal{P C}} d s \\
& +a_{5} \int_{0}^{\widehat{t}} e^{-\gamma_{3}(\widehat{t}-s)}\left\|y_{s}\right\|_{\mathcal{P C}} d s+\sum_{0<t \widehat{t}} c_{k} e^{-\gamma_{1}\left(\widehat{t}-t_{k}\right)} y\left(t_{k}^{-}\right) \\
& +\sum_{0<t<\widehat{t}} d_{k} e^{-\gamma_{1}\left(\widehat{t}-t_{k}\right)} y\left(t_{k}^{-}\right)+a_{6} \\
& \leq a_{1} e^{-\gamma_{1} \widehat{t}}+a_{2} e^{-\gamma_{2} \widehat{t}}+a_{3}\left[K e^{-\gamma \widehat{t}} e^{\gamma r}+\frac{a_{6}}{1-\kappa}\right] \\
& +a_{4} \int_{0}^{\widehat{t}} e^{-\gamma_{2}(\widehat{t}-s)}\left[K e^{-\gamma s} e^{\gamma r}+\frac{a_{6}}{1-\kappa}\right] d s \\
& +a_{5} \int_{0}^{\widehat{t}} e^{-\gamma_{3}(\hat{t}-s)}\left[K e^{-\gamma s} e^{\gamma r}+\frac{a_{6}}{1-\kappa}\right] d s \\
& +\sum_{0<t_{k}<\hat{t}} c_{k} e^{-\gamma_{1}\left(\widehat{t}-t_{k}\right)}\left[K e^{-\gamma t_{k}^{-}}+\frac{a_{6}}{1-\kappa}\right] \\
& +\sum_{0<t_{k}<\widehat{t}} d_{k} e^{-\gamma_{2}\left(\widehat{t}-t_{k}\right)}\left[K e^{-\gamma t_{k}^{-}}+\frac{a_{6}}{1-\kappa}\right] \\
& \leq\left(\frac{a_{1}+a_{2}}{K}+a_{3} e^{\gamma r}+\frac{a_{4} e^{\gamma r}}{\gamma_{2}-\gamma}+\frac{a_{5} e^{\gamma r}}{\gamma_{3}-\gamma}+\sum_{k=1}^{\infty} c_{k}+\sum_{k=1}^{\infty} d_{k}\right) K e^{-\gamma \widehat{t}} \\
& +\left(a_{3}+\frac{a_{4}}{\gamma_{2}}+\frac{a_{5}}{\gamma_{3}}+\sum_{k=1}^{\infty} c_{k}+\sum_{k=1}^{\infty} d_{k}\right) \frac{a_{6}}{1-\kappa}+a_{6}
\end{aligned}
$$

owing to Eqs. (3) and (4), it follows that

$$
y(t)<K e^{-\gamma \widehat{t}}+\frac{a_{6}}{1-\kappa},
$$

this contradicts the inequality (5). Hence the conclusion in Lemma 3.1 is proved.

Remark 3.2 It should be pointed out that when the $y\left(t_{k}^{-}\right)$of (2) changes to $y_{t_{k}^{-}}$, we only need to modify (4) to

$$
\frac{a_{1}+a_{2}}{K}+e^{\gamma r}\left(a_{3}+\frac{a_{4}}{\gamma_{2}-\gamma}+\frac{a_{5}}{\gamma_{3}-\gamma}+\sum_{k=1}^{\infty} c_{k}+\sum_{k=1}^{\infty} d_{k}\right)<1
$$

Lemma 3.1 still holds.

Remark 3.3 Incidentally, Lemma 3.1 contains and improves some recent results, e.g., Lemma 3.1 in [14], Lemma 3.3 in [17] and Lemma 3.1 in [19]. In other words, Lemma 3.1 
expands the application scope of impulsive-integral inequalities to study the global attracting set and exponential stability.

\section{Global attracting set and $p$ th moment exponential stability}

Before proceeding any further, we state two needed lemmas. Thanks to assumptions on $\mathcal{T}_{\alpha}(t)$, together with the techniques shown in Ref. [15], the proofs of the following two lemmas are straightforward and so will not be given.

Lemma 4.1 For any $\sigma:[0,+\infty] \rightarrow L_{Q}^{0}(\mathbb{K}, \mathbb{H})$ that satisfies condition $(H 4)$ and $\sup _{t \geq 0}\|\sigma(t)\|_{L_{Q}^{0}}<\infty$, there exists a constant $C>0$ depending on $\widetilde{M}, H, \gamma_{3}$ and $p$ such that

$$
\mathbb{E}\left\|\int_{0}^{t} \mathcal{T}_{\alpha}(t-s) \sigma(s) d B_{Q}^{H}(s)\right\|^{p} \leq C \sup _{t \geq 0}\|\sigma(t)\|_{L_{Q}^{0}}^{p}
$$

for any $p \geq 2$ and $t \geq 0$.

Lemma 4.2 If $\sigma:[0,+\infty] \rightarrow L_{Q}^{0}(\mathbb{K}, \mathbb{H})$ satisfies Hypothesis $(H 4)$ with

$$
\int_{0}^{+\infty} e^{\gamma_{3} s}\|\sigma(s)\|_{L_{Q}^{0}}^{2} d s<\infty
$$

then we have

$$
\mathbb{E}\left\|\int_{0}^{t} \mathcal{T}_{\alpha}(t-s) \sigma(s) d B_{Q}^{H}(s)\right\|^{p} \leq C e^{-\gamma_{3} t}
$$

for any $p \geq 2$ and $t \geq 0$, where the constant $C>0$ depends on $\widetilde{M}, H, p, \gamma_{3}$.

Then we will show and prove our main results in the next section.

Theorem 4.3 Let Hypotheses (H1)-(H5) and $\sup _{t \geq 0}\|\sigma(t)\|_{L_{Q}^{0}}<\infty$ hold, then $\mathbb{S}=\{x(t) \in$ $\left.\mathbb{H} \mid \mathbb{E}\|x(t)\|^{p} \leq(1-\widehat{\kappa})^{-1} \widehat{a}_{6}\right\}$ is the global attracting set of system (1) provided that

$$
\begin{aligned}
\widehat{\kappa}= & \delta+\frac{14^{p-1} \widetilde{M}^{p} \gamma_{2}^{-p} C_{f}^{p}}{(1-\delta)^{p-1}}+\frac{14^{p-1} \widetilde{M}^{p} \gamma_{3}^{-\frac{p}{2}} C_{h}^{p}\left(\frac{p(p-1)}{2}\right)^{\frac{p}{2}}\left(\frac{p-2}{2 p-2}\right)^{\frac{p}{2}-1}}{(1-\delta)^{p-1}} \\
& +\frac{7^{p-1} \widetilde{M}^{p}\left(\left(\sum_{k=1}^{\infty} c_{k}\right)^{\frac{p}{q}+1}+\left(\sum_{k=1}^{\infty} d_{k}\right)^{\frac{p}{q}+1}\right)}{(1-\delta)^{p-1}}<1,
\end{aligned}
$$

where $p \geq 2,1<q \leq 2$ with $\frac{1}{p}+\frac{1}{q}=1$, and

$$
\begin{aligned}
\widehat{a_{6}}= & \frac{14^{p-1} \widetilde{M}^{p} \gamma_{2}^{-p} l_{f}^{p}+14^{p-1} \widetilde{M}^{p} \gamma_{3}^{-\frac{p}{2}} l_{h}^{p}\left(\frac{p(p-1)}{2}\right)^{\frac{p}{2}}\left(\frac{p-2}{2 p-2}\right)^{\frac{p}{2}-1}}{(1-\delta)^{p-1}} \\
& +\frac{7^{p-1} C \sup _{t \geq 0}\|\sigma(t)\|_{L_{Q}^{0}}^{p}}{(1-\delta)^{p-1}}
\end{aligned}
$$


Proof It follows from Definition 2.6 that

$$
\begin{aligned}
\mathbb{E}\|x(t)\|^{p} \leq & \frac{1}{\delta^{p-1}} \mathbb{E}\left\|g\left(t, x_{t}\right)\right\|^{p}+\frac{1}{(1-\delta)^{p-1}} \mathbb{E}\left\|x(t)-g\left(t, x_{t}\right)\right\|^{p} \\
\leq & \frac{1}{\delta^{p-1}} \mathbb{E}\left\|g\left(t, x_{t}\right)\right\|^{p}+\frac{1}{(1-\delta)^{p-1}}\left\{14^{p-1} \mathbb{E}\left\|\mathcal{S}_{\alpha}(t) \psi(0)\right\|^{p}\right. \\
& +14^{p-1} \mathbb{E}\left\|\mathcal{S}_{\alpha}(t) g(0, \psi)\right\|^{p}+7^{p-1}\left\|\mathcal{K}_{\alpha}(t) \varphi\right\|^{p} \\
& +7^{p-1} \mathbb{E}\left\|\int_{0}^{t} \mathcal{K}_{\alpha}(t-s) f\left(s, x_{s}\right) d s\right\|^{p} \\
& +7^{p-1} \mathbb{E}\left\|\int_{0}^{t} \mathcal{T}_{\alpha}(t-s) h\left(s, x_{s}\right) d W(s)\right\|^{p} \\
& +7^{p-1} \mathbb{E}\left\|\int_{0}^{t} \mathcal{T}_{\alpha}(t-s) \sigma(s) d B_{Q}^{H}(s)\right\|^{p} \\
& +7^{p-1} \mathbb{E}\left\|\sum_{0<t_{k}<t} \mathcal{S}_{\alpha}\left(t-t_{k}\right) I_{k}\left(x\left(t_{k}^{-}\right)\right)\right\|^{p} \\
& \left.+7^{p-1} \mathbb{E}\left\|\sum_{0<t_{k}<t} \mathcal{K}_{\alpha}\left(t-t_{k}\right) G_{k}\left(x\left(t_{k}^{-}\right)\right)\right\|^{p}\right\} \\
\leq & \delta \mathbb{E}^{p}\left\|x_{t}\right\|_{\mathcal{P C}}^{p} \frac{14^{p-1}}{(1-\delta)^{p-1}\left(F_{1}+F_{2}\right)+\frac{7^{p-1}}{(1-\delta)^{p-1}} \sum_{j=3}^{8} F_{j} .}
\end{aligned}
$$

Now, we estimate each term on the right-hand side of the above formula one by one. In terms of Hypotheses $(H 1)$ and $(H 2)$, we get

$$
\begin{aligned}
& F_{1}+F_{2} \leq \tilde{M}^{p} e^{-p \gamma_{1} t} \mathbb{E}\|\psi(0)\|^{p}+\tilde{M}^{p} e^{-p \gamma_{1} t} \delta^{p} \mathbb{E}\|\psi\|_{\mathcal{P C}}^{p} \\
& \leq \tilde{M}^{p}\left(1+\delta^{p}\right) \mathbb{E}\|\psi\|_{\mathcal{P C}}^{p} e^{-\gamma_{1} t} \\
& F_{3} \leq \tilde{M}^{p} \mathbb{E}\|\varphi\|^{p} e^{-\gamma_{2} t} .
\end{aligned}
$$

By the aid of (H3) and Hölder's inequality, one can easily obtain

$$
\begin{aligned}
F_{4} & \leq \mathbb{E}\left(\int_{0}^{t} \tilde{M} e^{-\gamma_{2}(t-s)}\left(C_{f}\left\|x_{s}\right\|_{\mathcal{P C}}+\|f(s, 0)\|\right) d s\right)^{p} \\
& \leq 2^{p-1} \tilde{M}^{p} \gamma_{2}{ }^{1-p} C_{f}^{p} \int_{0}^{t} e^{-\gamma_{2}(t-s)} \mathbb{E}\left\|x_{s}\right\|_{\mathcal{P C}}^{p} d s+2^{p-1} \tilde{M}^{p} \gamma_{2}{ }^{-p} l_{f}^{p} .
\end{aligned}
$$

To proceed, the Hypothesis $(H 3)$ and Lemma 2.9 lead to

$$
\begin{aligned}
F_{5} \leq & \tilde{M}^{p}\left(\frac{p(p-1)}{2}\right)^{p / 2}\left(\int_{0}^{t}\left(e^{-p \gamma_{3}(t-s)} \mathbb{E}\left\|h\left(s, x_{s}\right)\right\|_{L_{2}^{0}}^{p}\right)^{2 / p} d s\right)^{p / 2} \\
= & \tilde{M}^{p}\left(\frac{p(p-1)}{2}\right)^{\frac{p}{2}}\left(\int_{0}^{t} e^{-\frac{2 \gamma_{3}(p-1)(t-s)}{p-2}} d s\right)^{\frac{p}{2}-1} \int_{0}^{t} e^{-\gamma_{3}(t-s)} \mathbb{E}\left\|h\left(s, x_{s}\right)\right\|_{L_{2}^{0}}^{p} d s \\
\leq & 2^{p-1} \tilde{M}^{p} C_{h}^{p}\left(\frac{p(p-1)}{2}\right)^{\frac{p}{2}}\left(\frac{p-2}{2 \gamma_{3}(p-1)}\right)^{\frac{p}{2}-1} \int_{0}^{t} e^{-\gamma_{3}(t-s)} \mathbb{E}\left\|x_{s}\right\|_{\mathcal{P C}}^{p} d s \\
& +2^{p-1} \tilde{M}^{p}\left(\frac{p(p-1)}{2}\right)^{\frac{p}{2}}\left(\frac{p-2}{2(p-1)}\right)^{\frac{p}{2}-1} \gamma_{3}^{-\frac{p}{2}} l_{h}^{p} .
\end{aligned}
$$


Thanks to Lemma 4.1, we directly obtain

$$
F_{6} \leq C \sup _{t \geq 0}\|\sigma(t)\|_{L_{Q}^{0}}^{p}
$$

For $1<q \leq 2$ with $\frac{1}{p}+\frac{1}{q}=1$, by utilizing condition (H5) and Hölder's inequality, we arrive at

$$
\begin{aligned}
F_{7} & \leq \mathbb{E}\left(\sum_{0<t_{k}}\left\|\mathcal{S}_{\alpha}\left(t-t_{k}\right)\right\|\left\|I_{k}\left(x\left(t_{k}^{-}\right)\right)\right\|\right)^{p} \\
& \leq \tilde{M}^{p} \mathbb{E}\left(\sum_{0<t_{k}<t} c_{k} e^{-\gamma_{1}\left(t-t_{k}\right)}\left\|x\left(t_{k}^{-}\right)\right\|\right)^{p} \\
& \leq \tilde{M}^{p}\left(\sum_{k=1}^{\infty} c_{k}\right)^{\frac{p}{q}} \sum_{0<t_{k}<t} c_{k} e^{-\gamma_{1}\left(t-t_{k}\right)} \mathbb{E}\left\|x\left(t_{k}^{-}\right)\right\|^{p},
\end{aligned}
$$

repeating the above computing for the term $F_{8}$, we deduce that

$$
\begin{aligned}
F_{8} & \leq \mathbb{E}\left(\sum_{0<t_{k}}\left\|\mathcal{K}_{\alpha}\left(t-t_{k}\right)\right\|\left\|G_{k}\left(x\left(t_{k}^{-}\right)\right)\right\|\right)^{p} \\
& \leq \tilde{M}^{p}\left(\sum_{k=1}^{\infty} d_{k}\right)^{\frac{p}{q}} \sum_{0<t_{k}<t} d_{k} e^{-\gamma_{2}\left(t-t_{k}\right)} \mathbb{E}\left\|x\left(t_{k}^{-}\right)\right\|^{p} .
\end{aligned}
$$

Putting (12)-(17) together into (11), we have

$$
\begin{aligned}
& \mathbb{E}\|x(t)\|^{p} \leq \delta \mathbb{E}\left\|x_{t}\right\|_{\mathcal{P C}}^{p}+\frac{14^{p-1}}{(1-\delta)^{p-1}}\left(\tilde{M}^{p}\left(1+\delta^{p}\right) \mathbb{E}\|\psi\|_{\mathcal{P C}}^{p} e^{-\gamma_{1} t}\right) \\
& +\frac{7^{p-1}}{(1-\delta)^{p-1}}\left\{\tilde{M}^{p} \mathbb{E}\|\varphi\|^{p} e^{-\gamma_{2} t}+2^{p-1} \tilde{M}^{p} \gamma_{2}^{-p} l_{f}^{p}\right. \\
& +2^{p-1} \tilde{M}^{p} \gamma_{2}{ }^{1-p} C_{f}^{p} \int_{0}^{t} e^{-\gamma_{2}(t-s)} \mathbb{E}\left\|x_{s}\right\|_{\mathcal{P C}}^{p} d s \\
& +2^{p-1} \tilde{M}^{p} C_{h}^{p}\left(\frac{p(p-1)}{2}\right)^{\frac{p}{2}}\left(\frac{p-2}{2 \gamma_{3}(p-1)}\right)^{\frac{p}{2}-1} \\
& \times \int_{0}^{t} e^{-\gamma_{3}(t-s)} \mathbb{E}\left\|x_{s}\right\|_{\mathcal{P C}}^{p} d s \\
& +2^{p-1} \tilde{M}^{p}\left(\frac{p(p-1)}{2}\right)^{\frac{p}{2}}\left(\frac{p-2}{2(p-1)}\right)^{\frac{p}{2}-1} \gamma_{3}^{-\frac{p}{2}} l_{h}^{p} \\
& +C \sup _{t \geq 0}\|\sigma(t)\|_{L_{Q}^{0}}^{p}+\tilde{M}^{p}\left(\sum_{k=1}^{\infty} c_{k}\right)^{\frac{p}{q}} \sum_{0<t_{k}<t} c_{k} e^{-\gamma_{1}\left(t-t_{k}\right)} \mathbb{E}\left\|x\left(t_{k}^{-}\right)\right\|^{p} \\
& \left.+\tilde{M}^{p}\left(\sum_{k=1}^{\infty} d_{k}\right)^{\frac{p}{q}} \sum_{0<t_{k}<t} d_{k} e^{-\gamma_{2}\left(t-t_{k}\right)} \mathbb{E}\left\|x\left(t_{k}^{-}\right)\right\|^{p}\right\} .
\end{aligned}
$$


Let $\widehat{a_{3}}=\delta$, and

$$
\begin{aligned}
\widehat{a_{1}}= & \frac{14^{p-1}}{(1-\delta)^{p-1}} \tilde{M}^{p}\left(1+\delta^{p}\right) \mathbb{E}\|\psi\|_{\mathcal{P C}}^{p}, \\
\widehat{a_{2}}= & \frac{7^{p-1}}{(1-\delta)^{p-1}} \tilde{M}^{p} \mathbb{E}\|\varphi\|^{p}, \\
\widehat{a_{4}}= & \frac{14^{p-1}}{(1-\delta)^{p-1}} \tilde{M}^{p} \gamma_{2}^{1-p} C_{f}^{p}, \\
\widehat{a_{5}}= & \frac{14^{p-1}}{(1-\delta)^{p-1}} \tilde{M}^{p} C_{h}^{p}\left(\frac{p(p-1)}{2}\right)^{\frac{p}{2}}\left(\frac{p-2}{2 \gamma_{3}(p-1)}\right)^{\frac{p}{2}-1}, \\
\widehat{a_{6}}= & \frac{14^{p-1} \tilde{M}^{p} \gamma_{2}^{-p} l_{f}^{p}+14^{p-1} \tilde{M}^{p} \gamma_{3}^{-\frac{p}{2}} l_{h}^{p}\left(\frac{p(p-1)}{2}\right)^{\frac{p}{2}}\left(\frac{p-2}{2 p-2}\right)^{\frac{p}{2}-1}}{(1-\delta)^{p-1}} \\
& +\frac{7^{p-1} C \sup _{t \geq 0}\|\sigma(t)\|_{L_{Q}^{0}}^{p}}{(1-\delta)^{p-1}}, \\
\widehat{c_{k}}= & \frac{7^{p-1} \widetilde{M}^{p}\left(\sum_{k=1}^{\infty} c_{k}\right)^{\frac{p}{q}} c_{k}}{(1-\delta)^{p-1}}, \quad \widehat{d}_{k}=\frac{7^{p-1} \widetilde{M}^{p}\left(\sum_{k=1}^{\infty} d_{k}\right)^{\frac{p}{q}} d_{k}}{(1-\delta)^{p-1}} .
\end{aligned}
$$

The inequality (10) implies that

$$
\widehat{a_{3}}+\frac{\widehat{a_{4}}}{\gamma_{2}}+\frac{\widehat{a_{5}}}{\gamma_{3}}+\sum_{k=1}^{\infty} \widehat{c_{k}}+\sum_{k=1}^{\infty} \widehat{d_{k}} \triangleq \widehat{\kappa}<1 .
$$

Since $\psi \in \mathcal{P C}_{\mathcal{F}_{0}}^{b}([-r, 0] ; \mathbb{H})$, there exists $\widehat{K} \geq\|\psi\|_{\mathcal{P C}}$ such that

$$
\frac{\widehat{a_{1}}+\widehat{a_{2}}}{\widehat{K}}+\widehat{a_{3}} e^{\gamma r}+\frac{\widehat{a_{4}} e^{\gamma r}}{\gamma_{2}-\gamma}+\frac{\widehat{a_{5}} e^{\gamma r}}{\gamma_{3}-\gamma}+\sum_{k=1}^{\infty} \widehat{c_{k}}+\sum_{k=1}^{\infty} \widehat{c_{k}}<1,
$$

with $\gamma \in(0, \gamma *), \gamma^{*}=\min \left\{\gamma_{1}, \gamma_{2}, \gamma_{3}\right\}$. Then it follows from Lemma 3.1 that

$$
\mathbb{E}\|x(t)\|^{p} \leq \widehat{K} e^{-\gamma t}+(1-\widehat{\kappa})^{-1} \widehat{a_{6}}
$$

Therefore, in view of Definition 2.7, we complete the proof of Theorem 4.3.

Theorem 4.4 Suppose that the hypotheses of Theorem 4.3 are satisfied with $l_{f}=l_{g}=0$,

$$
\int_{0}^{+\infty} e^{\gamma_{3} s}\|\sigma(s)\|_{L_{Q}^{0}}^{2} d s<\infty
$$

and

$$
\begin{aligned}
\widehat{\kappa}= & \delta+\frac{14^{p-1} \widetilde{M}^{p} \gamma_{2}^{-p} C_{f}^{p}}{(1-\delta)^{p-1}}+\frac{14^{p-1} \tilde{M}^{p} \gamma_{3}^{-\frac{p}{2}} C_{h}^{p}\left(\frac{p(p-1)}{2}\right)^{\frac{p}{2}}\left(\frac{p-2}{2 p-2}\right)^{\frac{p}{2}-1}}{(1-\delta)^{p-1}} \\
& +\frac{7^{p-1} \tilde{M}^{p}\left(\left(\sum_{k=1}^{\infty} c_{k}\right)^{\frac{p}{q}+1}+\left(\sum_{k=1}^{\infty} d_{k}\right)^{\frac{p}{q}+1}\right)}{(1-\delta)^{p-1}}<1 .
\end{aligned}
$$


Then the mild solution of system (1) is $p$ th moment exponentially stable.

Proceeding as in the proof of Theorem 4.3, by virtue of Lemmas 4.2 and 3.1, one can verify Theorem 4.4 holds. We omit details of the proof here.

At the end of this part, some pertinent results are stated without proof.

If the neutral term $g(t, \cdot)=0$, the system (1) reduces to the following impulsive fractional stochastic partial functional integro-differential equation:

$$
\left\{\begin{aligned}
&{ }^{c} D_{t}^{\alpha} x(t)= A x(t)+\mathbb{I}_{t}^{2-\alpha}\left(f\left(t, x_{t}\right)\right)+h\left(t, x_{t}\right) \frac{d W(t)}{d t} \\
&+\sigma(t) \frac{d B_{Q}^{H}(t)}{d t}, \quad t \neq t_{k}, t \in J, \\
& \Delta x\left(t_{k}\right)=I_{k}\left(x\left(t_{k}^{-}\right)\right), \quad \Delta x^{\prime}\left(t_{k}\right)=G_{k}\left(x\left(t_{k}^{-}\right)\right), \quad t=t_{k}, k=1,2, \ldots, \\
& x(t)=\psi(t) \in \mathcal{P C}_{\mathcal{F}_{0}}^{b}([-r, 0] ; \mathbb{H}), \quad t \in[-r, 0], \\
& x^{\prime}(0)=\varphi \in \mathbb{H} .
\end{aligned}\right.
$$

Corollary 4.5 Assume that Hypotheses (H1), (H3)-(H5) with $l_{f}=l_{g}=0$ and $\int_{0}^{+\infty} e^{\gamma_{3} s} \times$ $\|\sigma(s)\|_{L_{Q}^{0}}^{2} d s<\infty$ are satisfied. If for any $p \geq 2,1<q \leq 2$ with $\frac{1}{p}+\frac{1}{q}=1$,

$$
\begin{aligned}
\widehat{\kappa}= & 7^{p-1} \widetilde{M}^{p} \gamma_{2}^{-p} C_{f}^{p}+7^{p-1} \tilde{M}^{p} \gamma_{3}^{-\frac{p}{2}} C_{h}^{p}\left(\frac{p(p-1)}{2}\right)^{\frac{p}{2}}\left(\frac{p-2}{2 p-2}\right)^{\frac{p}{2}-1} \\
& +7^{p-1}\left(\sum_{k=1}^{\infty} c_{k}\right)^{\frac{p}{q}+1}+7^{p-1}\left(\sum_{k=1}^{\infty} d_{k}\right)^{\frac{p}{q}+1}<1
\end{aligned}
$$

then the mild solution to system (20) is exponentially stable in pth moment.

When $\Delta x\left(t_{k}\right), \Delta x^{\prime}\left(t_{k}\right)=0, k=1,2, \ldots$, the system (1) degenerates to fractional neutral stochastic partial functional integro-differential equation without impulses as follows:

$$
\left\{\begin{aligned}
&{ }^{c} D_{t}^{\alpha}\left[x(t)-g\left(t, x_{t}\right)\right]= A\left[x(t)-g\left(t, x_{t}\right)\right]+\mathbb{I}_{t}^{2-\alpha}\left(f\left(t, x_{t}\right)\right) \\
&+h\left(t, x_{t}\right) \frac{d W(t)}{d t}+\sigma(t) \frac{d B_{Q}^{H}(t)}{d t}, \quad t \in J, \\
& x(t)=\psi(t) \in \mathcal{P} \mathcal{C}_{\mathcal{F}_{0}}^{b}([-r, 0] ; \mathbb{H}), \quad t \in[-r, 0], \\
& \frac{d}{d t}\left[x(t)-g\left(t, x_{t}\right)\right]_{t=0}=\varphi \in \mathbb{H} .
\end{aligned}\right.
$$

Corollary 4.6 Suppose that assumptions (H1), (a) of (H2), (H3) and (H4) with $l_{f}=l_{g}=0$, $\int_{0}^{+\infty} e^{\gamma_{3} s}\|\sigma(s)\|_{L_{Q}^{0}}^{2} d s<\infty$ and the inequality

$$
10^{p-1}\left(\tilde{M}^{p} \gamma_{2}^{-p} C_{f}^{p}+\widetilde{M}^{p} \gamma_{3}^{-\frac{p}{2}} C_{h}^{p}\left(\frac{p(p-1)}{2}\right)^{\frac{p}{2}}\left(\frac{p-2}{2 p-2}\right)^{\frac{p}{2}-1}\right)<(1-\delta)^{p}
$$

hold, then the mild solution of system (21) is pth moment exponentially stable. 
If the neutral term $g(t, \cdot)=0$ and $\Delta x\left(t_{k}\right), \Delta x^{\prime}\left(t_{k}\right)=0, k=1,2, \ldots$, the system (1) degrades to fractional stochastic partial functional integro-differential equation:

$$
\left\{\begin{array}{l}
{ }^{c} D_{t}^{\alpha} x(t)=A x(t)+\mathbb{I}_{t}^{2-\alpha}\left(f\left(t, x_{t}\right)\right)+h\left(t, x_{t}\right) \frac{d W(t)}{d t} \\
\quad+\sigma(t) \frac{d B_{Q}^{H}(t)}{d t}, \quad t \in J, \\
x(t)=\psi(t) \in \mathcal{P C}_{\mathcal{F}_{0}}^{b}([-r, 0] ; \mathbb{H}), \quad t \in[-r, 0], \\
x^{\prime}(0)=\varphi \in \mathbb{H} .
\end{array}\right.
$$

Corollary 4.7 Let us assume that conditions $(H 1),(H 3),(H 4)$ with $l_{f}=l_{g}=0, \int_{0}^{+\infty} e^{\gamma_{3} s} \times$ $\|\sigma(s)\|_{L_{Q}^{0}}^{2} d s<\infty$ and the following inequality:

$$
5^{p-1} \tilde{M}^{p}\left(\gamma_{2}^{-p} C_{f}^{p}+\gamma_{3}^{-\frac{p}{2}} C_{h}^{p}\left(\frac{p(p-1)}{2}\right)^{\frac{p}{2}}\left(\frac{p-2}{2 p-2}\right)^{\frac{p}{2}-1}\right)<1
$$

hold, then the mild solution for system (22) is pth moment exponentially stable. In particular, when $\gamma_{2}^{2}>5 \tilde{M}^{2} C_{f}^{2}$, the mild solution of system (22) is exponentially stable in mean square.

Remark 4.8 Thanks to Remark 3.2, our approach in this paper is applicable for FNSEEs involving delayed impulses:

$$
\left\{\begin{aligned}
&{ }^{c} D_{t}^{\alpha}\left[x(t)-g\left(t, x_{t}\right)\right]= A\left[x(t)-g\left(t, x_{t}\right)\right]+\mathbb{I}_{t}^{2-\alpha}\left(f\left(t, x_{t}\right)\right) \\
&+h\left(t, x_{t}\right) \frac{d W(t)}{d t}+\sigma(t) \frac{d B_{Q}^{H}(t)}{d t}, \quad t \neq t_{k}, t \in J, \\
& \Delta x\left(t_{k}\right)=I_{k}\left(x_{t_{k}^{-}}\right), \quad \Delta x^{\prime}\left(t_{k}\right)=G_{k}\left(x_{t_{k}^{-}}\right), \quad t=t_{k}, k=1,2, \ldots, \\
& x(t)=\psi(t) \in \mathcal{P} \mathcal{C}_{\mathcal{F}_{0}}^{b}([-r, 0] ; \mathbb{H}), \quad t \in[-r, 0], \\
& \frac{d}{d t}\left[x(t)-g\left(t, x_{t}\right)\right]_{t=0}=\varphi \in \mathbb{H},
\end{aligned}\right.
$$

i.e., our results can be easily extended to FNSEEs with delayed impulses.

\section{Example}

Example 5.1 We consider the following impulsive fractional neutral stochastic integrodifferential equation:

$$
\left\{\begin{array}{l}
\frac{\partial^{\alpha}\left[x(t)-\beta_{1} x_{t}\right]}{\partial t^{\alpha}}=\frac{\partial^{2}}{\partial z^{2}}\left[x(t)-\beta_{1} x_{t}\right]+\mathbb{I}_{t}^{2-\alpha}\left(\beta_{2} x_{t}+\chi_{2}\right)+\left(\beta_{3} x_{t}+\chi_{2}\right) \frac{d W(t)}{d t} \\
\quad+\sigma(t) \frac{d B_{Q}^{H}(t)}{d t}, \quad t \geq 0, t \neq t_{k}, 0 \leq z \leq \pi, \\
\Delta x\left(t_{k}\right)=\frac{\beta_{4}}{k^{2}} x\left(t_{k}^{-}\right), \quad \Delta x^{\prime}\left(t_{k}\right)=\frac{\beta_{5}}{k^{2}} x\left(t_{k}^{-}\right), \quad t=t_{k}, k=1,2, \ldots, \\
x(t, 0)=x(t, \pi)=0, \\
x(t)=\psi(t) \in \mathcal{P C}_{\mathcal{F}_{0}}^{b}\left([-r, 0] ; L^{2}[0, \pi]\right), \quad t \in[-r, 0], \\
\frac{d}{d t}\left[x(t)-\beta_{1} x_{t}\right]_{t=0}=\varphi \in L^{2}[0, \pi],
\end{array}\right.
$$

where $\beta_{i}, i=1,2, \ldots, 5, \chi_{j}, j=1,2$ are positive constants, $W(t)$ is a Wiener process, $\sigma(t)$ is a continuous function satisfying Hypothesis $(H 4)$ with $\sup _{t \geq 0}\|\sigma(t)\|_{L_{Q}^{0}}<\infty$, and $B_{Q}^{H}(t)$ is a 
fractional Brownian motion. Define $\mathbb{H}=L^{2}[0, \pi], A=\frac{\partial^{2}}{\partial z^{2}}$ and $\mathcal{D}(A)=\mathbb{H}_{0}^{1}(0, \pi) \cap \mathbb{H}^{2}(0, \pi)$. Then

$$
A w=-\sum_{n=1}^{\infty} n^{2}\left\langle w, e_{n}(z)\right\rangle e_{n}(z), \quad w \in \mathcal{D}(A)
$$

where $e_{n}(z)=\sqrt{\frac{2}{\pi}} \sin (n z), 0 \leq z \leq \pi, n \in \mathbb{N}$. It is well known that $A$ is the infinitesimal generator of a strongly continuous semigroup of bounded linear operators $T(t): \mathbb{H} \rightarrow \mathbb{H}$ and it is given by

$$
T(t) w=\sum_{n=1}^{\infty} e^{-n^{2} t}\left\langle w, e_{n}(z)\right| e_{n}(z), \quad w \in \mathbb{H} \quad \text { and } \quad\|T(t)\| \leq e^{-\pi^{2} t}
$$

Based on Ref. [26], then we can define the solution operators $\mathcal{S}_{\alpha}(t), \mathcal{K}_{\alpha}(t), \mathcal{T}_{\alpha}(t)$ such that $\left\|\mathcal{S}_{\alpha}(t)\right\| \leq e^{-\gamma_{1} t},\left\|\mathcal{K}_{\alpha}(t)\right\| \leq e^{-\gamma_{2} t},\left\|\mathcal{T}_{\alpha}(t)\right\| \leq e^{-\gamma_{3} t}$ with $\widetilde{M}=1$, where $\gamma_{1}, \gamma_{2}, \gamma_{3}<\pi^{2}$.

Fix $\beta=3 / 4$, from the definition of $(-A)^{-3 / 4}$ [29], we have $\left\|(-A)^{-3 / 4}\right\| \leq \frac{1}{\pi^{3 / 2}}$ and $\left\|(-A)^{3 / 4}\right\|=1$.

Let $g\left(t, x_{t}\right)=\beta_{1} x_{t}, f\left(t, x_{t}\right)=\beta_{2} x_{t}+\chi_{1}, h\left(t, x_{t}\right)=\beta_{3} x_{t}+\chi_{2}$.

Then Hypotheses (H2), (H3) and (H5) are satisfied with

$$
\begin{aligned}
& \delta=\left\|(-A)^{-\beta}\right\| \beta_{1}=\frac{\beta_{1}}{\pi^{3 / 2}}, \quad C_{f}=\beta_{2}, \quad C_{h}=\beta_{3}, \\
& l_{f}=\chi_{1}, \quad l_{h}=\chi_{2}, \quad c_{k}=\frac{\beta_{4}}{k^{2}}, \quad d_{k}=\frac{\beta_{5}}{k^{2}} .
\end{aligned}
$$

Let $p=2$, then we have

$$
\begin{aligned}
\widehat{\kappa} & =\delta+\frac{14 \gamma_{2}^{-2} \beta_{2}^{2}}{1-\delta}+\frac{14 \gamma_{3}^{-1} \beta_{3}^{2}}{1-\delta}+\frac{7\left(\left(\sum_{k=1}^{\infty} \frac{\beta_{4}}{k^{2}}\right)^{2}+\left(\sum_{k=1}^{\infty} \frac{\beta_{5}}{k^{2}}\right)^{2}\right)}{1-\delta} \\
& =\frac{\beta_{1}}{\pi^{3 / 2}}+\frac{14 \gamma_{2}^{-2} \beta_{2}^{2}}{1-\frac{\beta_{1}}{\pi^{3 / 2}}}+\frac{14 \gamma_{3}^{-1} \beta_{3}^{2}}{1-\frac{\beta_{1}}{\pi^{3 / 2}}}+\frac{7 \pi^{4}\left(\beta_{4}^{2}+\beta_{5}^{2}\right)}{36\left(1-\frac{\beta_{1}}{\pi^{3 / 2}}\right)}=: \widehat{\kappa}_{0} \\
\widehat{a}_{6} & =\frac{14 \gamma_{2}^{-2} \chi_{1}^{2}+14 \gamma_{3}^{-1} \chi_{2}^{2}+7 C \sup _{t \geq 0}\|\sigma(t)\|_{L_{Q}^{0}}^{2}}{1-\frac{\beta_{1}}{\pi^{3 / 2}}} .
\end{aligned}
$$

From Theorem 4.3, we conclude that $\mathbb{S}=\left\{x(t) \in \mathbb{H} \mid \mathbb{E}\|x(t)\|^{2} \leq\left(1-\widehat{\kappa}_{0}\right)^{-1} \widehat{a}_{6}\right\}$ is the global attracting set of system (24) provided that $\widehat{\kappa}_{0}<1$. In particular, when $\chi_{1}=\chi_{2}=0$ and $\widehat{\kappa}_{0}<1$, by Theorem 4.4, the mild solution of system (24) is exponentially stable in mean square.

\section{Conclusion}

In this paper, by establishing a new impulsive-integral inequality, we obtain the global attracting sets and some sufficient conditions which guarantee the $p$ th moment exponential stability of mild solutions for impulsive fractional neutral stochastic integro-differential equations driven by $\mathrm{fBm}$ and standard $\mathrm{Bm}$. And by a suitable modification to our proposed inequality, we show that it is applicable for neutral stochastic differential equations with delayed impulses. In our future work, we will consider the following three issues. Firstly, we will discuss the existence, uniqueness, exponential stability and approximate controllability of the system (1) with non-instantaneous impulses. Secondly, we will explore the global 
attracting sets and exponential stability for another kind of fractional neutral stochastic integro-differential equations driven by fBm, like the system in Ref. [9]. Thirdly, in view of the research development of infinite dimension G-Brownian motion and infinite dimension stochastic calculus in $G$-framework, we will investigate the global attracting sets and exponential stability of impulsive neutral stochastic partial functional integro-differential equations driven by G-Brownian motion.

Acknowledgements

The authors gratefully acknowledge the helpful comments of the editors and the anonymous reviewers.

Funding

We thank the support of the National Natural Science Foundation of China (Grant Nos. 11872305, 11532011) for our work.

\section{Availability of data and materials}

Data sharing not applicable to this article as no datasets were generated or analysed during the current study.

\section{Competing interests}

The authors declare that they have no competing interests.

\section{Authors' contributions}

All authors read and approved the final manuscript.

\section{Author details}

'Department of Applied Mathematics, Northwestern Polytechnical University, Xi'an, China. ${ }^{2}$ School of Applied Science, Taiyuan University of Science and Technology, Taiyuan, China.

\section{Publisher's Note}

Springer Nature remains neutral with regard to jurisdictional claims in published maps and institutional affiliations.

Received: 21 October 2019 Accepted: 15 January 2020 Published online: 07 February 2020

\section{References}

1. Kilbas, A.A., Srivastava, H.M., Trujillo, J.J. Theory and Applications of Fractional Differential Equations. Elsevier, Amsterdam (2006)

2. Sakthivel, R., Suganya, S., Anthoni, S.M.: Approximate controllability of fractional stochastic evolution equations. Comput. Math. Appl. 63(3), 660-668 (2012)

3. Sakthivel, R., Revathi, P., Ren, Y.: Existence of solutions for nonlinear fractional stochastic differential equations. Nonlinear Anal. 81, 70-86 (2013)

4. Wang, J.R.: Approximate mild solutions of fractional stochastic evolution equations in Hilbert spaces. Appl. Math. Comput. 256, 315-323 (2011)

5. Lakshmikantham, V., Bainov, V.D., Simeonov, P.S.: Theory of Impulsive Differential Equations. World Scientific Singapore (1989)

6. Benchohra, M., Henderson, J., Ntouyas, S.: Impulsive Differential Equations and Inclusions. Hindawi Publishing Corporation, New York (2006)

7. Ren, Y., Jia, X.J., Sakthivel, R.: The $p$-th moment stability of solutions to impulsive stochastic differential equations driven by G-Brownian motion. Appl. Anal. 96(6), 988-1003 (2017)

8. Ren, Y., He, Q., Gu, Y.F., Sakthivel, R.: Mean-square stability of delayed stochastic neural networks with impulsive effects driven by G-Brownian motion. Stat. Probab. Lett. 143, 56-66 (2018)

9. Yan, Z., Zhang, H.: Asymptotic stability of fractional impulsive neutral stochastic partial integro-differential equations with state-dependent delay. Electron. J. Differ. Equ. 2013, 206 (2013)

10. Zang, Y., Li, J.: Approximate controllability of fractional impulsive neutral stochastic differential equations with nonlocal conditions. Bound. Value Probl. 2013(1), 193 (2013)

11. Chadha, A., Pandey, D.N.: Existence results for an impulsive neutral stochastic fractional integro-differential equation with infinite delay. Nonlinear Anal. 128, 149-175 (2015)

12. Ren, Y., Yin, W.S., Sakthivel, R.: Stabilization of stochastic differential equations driven by G-Brownian motion with feedback control based on discrete-time state observation. Automatica 95, 146-151 (2018)

13. Chen, H.: Impulsive-integral inequality and exponential stability for stochastic partial differential equations with delays. Stat. Probab. Lett. 80(1), 50-56 (2010)

14. Long, S., Teng, L., Xu, D.: Global attracting set and stability of stochastic neutral partial functional differential equations with impulses. Stat. Probab. Lett. 82(9), 1699-1709 (2012)

15. Li, Z: Global attracting and quasi-invariant sets of impulses neutral stochastic functional differential equations driven by fBm. Neurocomputing 117, 620-627 (2016)

16. Liu, K., Li, Z.: Global attracting set, exponential decay and stability in distribution of neutral SPDEs driven by additive $\alpha$-stable processes. Discrete Contin. Dyn. Syst., Ser. B 21(10), 3551-3573 (2016)

17. Duan, P., Ren, Y.: Attracting and quasi-invariant sets of neutral stochastic integro-differential equations with impulses driven by fractional Brownian motion. Adv. Differ. Equ. 2017(1), 361 (2017) 
18. Sakthivel, R., Revathi, P., Ren, Y., Shen, G.J.: Retarded stochastic differential equations with infinite delay driven by Rosenblatt process. Stoch. Anal. Appl. 36(2), 304-323 (2018)

19. Xu, L., Li, Z., Luo, J.: Global attracting set and exponential decay of second-order neutral stochastic functional differential equations driven by fBm. Adv. Differ. Equ. 2017(1), 134 (2017)

20. Deng, S.F., Shu, X.B., Mao, J.Z.: Existence and exponential stability for impulsive neutral stochastic functional differential equations driven by fBm with noncompact semigroup via Mönch fixed point. J. Math. Anal. Appl. 467(1) 398-420 (2018)

21. $\mathrm{Xu}, \mathrm{L} ., \mathrm{Luo}, \mathrm{J}$.: Global attractiveness and exponential decay of neutral stochastic functional differential equations driven by fBm with Hurst parameter less than 1/2. Front. Math. China 13(6), 1469-1487 (2018)

22. Cheng, P., Deng, F., Yao, F.: Exponential stability analysis of impulsive stochastic functional differential systems with delayed impulses. Commun. Nonlinear Sci. Numer. Simul. 19(6), 2104-2114 (2014)

23. Biagini, F., Hu, Y., Øksendal, B., Zhang, T.: Stochastic Calculus for Fractional Brownian Motion and Applications. Springer, Berlin (2008)

24. Caraballo, T., Garrido-Atienza, M.J., Taniguchi, T.: The existence and exponential behavior of solutions to stochastic delay evolution equations with a fractional Brownian motion. Nonlinear Anal., Theory Methods Appl. 74(11), 3671-3684 (2011)

25. Shu, X., Wang, Q.: The existence and uniqueness of mild solutions for fractional differential equations with nonlocal conditions of order $1<\alpha<2$. Comput. Math. Appl. 64(6), 2100-2110 (2012)

26. Shu, X., Shi, Y: A study on the mild solution of impulsive fractional evolution equations. Appl. Math. Comput. 273, 465-476 (2016)

27. Chadha, A., Bora, S.N.: Existence and exponential stability for neutral stochastic fractional differential equations with impulses driven by Poisson jumps. Stochastics 90(5), 663-681 (2018)

28. Da Prato, G., Zabczyk, J.: Stochastic Equations in Infinite Dimensions. Cambridge University Press, Cambridge (2014)

29. Pazy, A.: Semigroups of Linear Operators and Applications to Partial Differential Equations. Springer, Berlin (2012)

\section{Submit your manuscript to a SpringerOpen ${ }^{\circ}$ journal and benefit from:}

- Convenient online submission

- Rigorous peer review

- Open access: articles freely available online

- High visibility within the field

- Retaining the copyright to your article

Submit your next manuscript at $\gg$ springeropen.com 